Recepción: 20 / 12 / 2017

Aceptación: 15 / 02 / 2018

Publicación: 8 / 05 / 2018

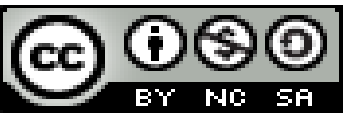

\title{
Estilo de vida factor predisponente del consumo de alcohol en la adolescencia
}

\author{
Lifestyle predisposing factor of the consumption alcohol in adolescence
}

Fator predisponente ao estilo de vida do consumo de álcool na adolescência

\author{
Marisela M. Saltos-Solís I \\ mariselamil@hotmail.com \\ Gabriel A. Rivera-Saltos II \\ gabriel_rivera1995@outlook.com
}

Correspondencia: marisela.saltoss@ug.edu.ec

I. Magister en Salud Pública, Magister en Gerencia Educativa, Especialista en Gestión Educativa, Universidad de Guayaquil. Guayaquil, Ecuador.

II. Estudiante del 10mo nivel de la Carrera de Medicina, Universidad Católica Santiago de Guayaquil, Guayaquil. Ecuador. 


\section{Resumen}

El objetivo de esta investigación fue determinar como el estilo de vida de la población adolescente según la edad y el género se relaciona con el consumo de alcohol. Metodología: estudio no experimental, observacional, descriptivo y correlacional, se realizó con 2351 estudiantes de ambos sexos del primero, segundo y tercer año de bachillerato de tres colegios fiscales del área urbana del cantón Milagro. Se midió el nivel de consumo de alcohol según edad y género, variables del estilo de vida, percepción y características del consumo. Resultados: el 39,63\% son hombres y el 60,37\% mujeres, con un 97,9\% de estudiantes mestizos. La media de edad del consumo de alcohol es de 16 años. No existe diferencia significativa en el consumo de alcohol por género. En relación al número de días el sexo masculino presenta un mayor nivel de ingesta de alcohol que en las mujeres. Conclusiones: los/as adolescentes tienen hábitos de vida no saludables, asociados a la escasa práctica de ejercicios físicos, actividades de recreación, ingieren en mayor proporción comidas rápidas evidenciándose hábitos alimentarios nocivos que incrementan el riesgo para la salud.

Palabras clave: Alcohol, estilo de vida, adolescentes, edad, género, consumo. 


\section{Abstract}

The objective of this research was to determine how the lifestyle of the adolescent population according to age and gender is related to alcohol consumption. Methodology: non-experimental, observational, descriptive and correlational study was carried out with 2351 students of both sexes of the first, second and third year of high school of three fiscal colleges of the urban area of the Milagro canton. The level of alcohol consumption was measured according to age and gender, lifestyle variables, perception and consumption characteristics. Results: 39.63\% are men and $60.37 \%$ are women, with $97.9 \%$ of mestizo students. The average age of alcohol consumption is 16 years. There is no significant difference in alcohol consumption by gender. In relation to the number of days, the male sex has a higher level of alcohol intake than in women. Conclusions: adolescents have unhealthy life habits, associated with the scarce practice of physical exercises, recreational activities, eat a greater proportion of fast foods evidencing harmful eating habits that increase the risk to health.

Keywords: Alcohol, tobacco, lifestyle, teenagers, age, gender, consumption. 


\section{Introducción.}

El consumo de alcohol es uno de los principales problemas de salud pública que se presentan en todo el mundo; la Organización Mundial de la Salud estima que 3.3 millones de personas mueren cada año a causa de su uso, esto representa el 5.9\% de las defunciones de forma global, a lo cual se le añade que es causante de múltiples enfermedades y trastornos mentales, origina discapacidad, pérdidas sociales y económicas, que se presentan cada vez a edades más tempranas (OMS, 2014).

Tegoma Ruiz y Cortaza Ramírez (2016) señalan que para América Latina, el alcohol es la puerta de entrada para el consumo de otras drogas en jóvenes y adolescentes; el consumo se favorece por la facilidad con que adquieren este producto debido a la falta de cumplimiento de las leyes establecidas para su compra y venta.

El consumo de sustancias adictivas como el alcohol y tabaco son un problema de Salud Pública que afecta a la población (Chelet, Escriche, García y Moreno, 2011; Organización Mundial de la Salud, 2014). Se produce desde edad temprana y tiene implicaciones en el ámbito social, educativo, personal, de salud, y actúa sin distinción de edad, sexo, clase social u ocupación.

Según el Instituto Nacional de Estadísticas y Censos (2012), el consumo de alcohol se inicia en edad temprana, la mayoría se encuentra en edades de 19 a 24 años, el $12 \%$ de la población de este grupo adquirió alguna bebida alcohólica. La edad promedio de inicio de consumo de bebidas alcohólicas es de 13,7 años (OPS, 2012).

La adolescencia es una etapa de la vida donde el riesgo es mayor porque se generan consecuencias negativas a nivel biológico, físico, emocional y psicológico (Cicua, Méndez y Muñoz, 2008). Es un período de grandes cambios, caracterizado por nuevos hábitos y exposición a 
factores de riesgo como tabaquismo, dieta inadecuada, sedentarismo (Carvalho, Medeiros, López, Barreto y Morais, 2014). En la transición de la niñez a la adolescencia es donde se experimenta con alcohol y otras drogas (Malta, Porto, Melo, Monteiro, Sardinha y Lessa, 2011).

Salazar y Arrivillaga (2004) señalan que los estilos de vida relacionados con el consumo de drogas incluyen comportamientos propios del consumo, tales como el modo de empleo de la droga y la frecuencia con que se usa, y el manejo de los efectos del consumo tanto a nivel personal (alteraciones físicas, cognoscitivas, emocionales y conductuales) como social (manejo de las relaciones interpersonales, pertenencia a grupos).

El tener un estilo de vida, saludable o no saludable, como hábitos nocivos, hábitos alimenticios, actividades físicas, vida sedentaria, relaciones sexuales a edad temprana, manejo del tiempo libre, producido en este proceso de cambio es un factor de riesgo o de protección en el ingreso al uso de drogas lícitas (Huescar, Cervello, Llamas y Moreno, 2011). Estudios como el de Balaguer (2002) manifiestan que la práctica de la actividad física es una conducta del adolescente que favorece el desarrollo de estilos de vida saludables. En tanto que los hábitos alimentarios nocivos se presentan como un elemento de riesgo para la salud (Jiménez, 2011).

El conjunto de pautas y hábitos que son cotidianos y demuestran cierta consistencia en el tiempo, bajo condiciones más o menos constantes se constituyen en factores de riesgo o de protección para la salud (Henderson, Hall y Lipton, 1980). Para Tirado, Aguaded y Marín (2009) la necesidad de experimentar nuevas sensaciones, adaptarse al mundo social, combatir el aburrimiento, ocio, son factores de riesgo del consumo de drogas. Los adolescentes al enfrentarse a situaciones de riesgo en la búsqueda de su identidad y personalidad consumen sustancias, especialmente por la presión e influencia de los iguales (Ruiz y Ruiz, 2011). 
Peñafiel (2009) manifiesta que los factores de riesgo son una característica interna y/o externa al individuo cuya presencia aumenta la probabilidad o la predisposición de que se produzca un determinado fenómeno como el consumo de alcohol y tabaco. Los adolescentes perciben que existe un menor riesgo para la salud con el consumo de drogas legales que en las ilegales pero la frecuencia de su uso es perjudicial para la salud. (Álvarez, et al., 2005: 16).

Autores como Ariza y Nebot (2002) y; Muñoz-Rivas y Graña (2001) manifiestan se debe considerar que la edad aumenta la frecuencia e intensidad del consumo de drogas, los 13 y 15 años es una edad vulnerable por los cambios fisiológicos, psicológicos y socioculturales que se producen en la adolescencia.

La Organización Mundial de la Salud (OMS) en su Sistema de Información Global sobre Alcohol y Salud, año 2014, expone que se consumió en el mundo 6.2 litros de alcohol por personas de 15 años y más, así como 60 tipos de enfermedades son ocasionadas por éste. De acuerdo a la Cuarta encuesta nacional sobre uso de drogas (2012) la influencia del contexto social, la cultura orientada al consumo, la baja percepción de los riesgos, influyen en el incremento de casos de uso de sustancias como el alcohol y tabaco.

El alcohol es un factor facilitador de las relaciones sociales en los adolescentes, lo cual explicaría que el grupo de amigos sea el principal iniciador en el hábito de beber y también que tener amigos bebedores sea un factor asociado al consumo de alcohol en el adolescente (Paniagua, García, Castellano, Sarrallé y Redondo, 2001).

El consumo de alcohol generalmente está relacionado con estilos de vida de riesgo como: consumo de tabaco y uso de drogas ilegales; trastornos mentales, como la depresión y la ansiedad; desórdenes alimenticios y aumento del índice de masa corporal; y, también, las peleas en la escuela, 
acoso, vandalismo y otros tipos de violencia. (Malta, Sardinha, Mendes, Barreto, Giatti, Castro, et al., 2009).

El alcohol es la droga más consumida por los adolescentes y presenta un patrón de consumo esporádico, intensivo, fuera del hogar y con sus pares (Paniagua, García, et al, 2001). La conducta desinhibida que induce el consumo de alcohol y de drogas ilegales facilita las relaciones sexuales precoces y en consecuencia las enfermedades de transmisión sexual y los embarazos no deseados (Miret, Rodes, Valverde, Geli y Cassabona, 1997).

De acuerdo con el informe de la OMS (2014), en América Latina lo que más se ingiere es cerveza (53\%); seguida de un 32,6\% de licores (vodka, whisky), y un 11,7\% de vino. En Ecuador las cifras también son lideradas por la cerveza con el $67 \%$, los licores con un $32 \%$ y el vino con el $1 \%$.

El alcohol afecta a las personas y las sociedades de diferentes maneras, y sus efectos están determinados por el volumen de alcohol consumido, los hábitos de consumo y, en raras ocasiones, la calidad del alcohol. En 2012, unos 3,3 millones de defunciones, o sea el 5,9\% del total mundial, fueron atribuibles al consumo de alcohol (OMS, 2014).

El consumo nocivo de alcohol también puede perjudicar a otras personas, por ejemplo, familiares, amigos, compañeros de trabajo y desconocidos. Asimismo, el consumo nocivo de alcohol genera una carga sanitaria, social y económica considerable para el conjunto de la sociedad

El propósito de la investigación fue el conocer en qué medida el estilo de vida de los adolescentes según la edad y el género se relaciona con el consumo de alcohol. 


\section{Metodología.}

El presente estudio es una investigación no experimental, observacional, descriptiva y correlacional, que se realizó con 2351 estudiantes de ambos sexos de tres colegios físcales del área urbana del cantón Milagro, del primero, segundo y tercer año de bachillerato, cuyas edades oscilaron entre los 13 a 25 años, con un promedio de edad de 17.

Se accedió a fuentes primarias de información, mediante la Encuesta Mundial de Salud a escolares, (GSHS), instrumento validado por la Organización Mundial de la Salud (2008), aplicado en diversos países de las regiones de América, África, Mediterráneo, Europa, Pacífico Occidental, Asia Occidental. El cuestionario permitió medir a) el nivel de consumo de alcohol y tabaco según la edad y el género, b) identificar las variables del estilo de vida relacionadas al consumo, b) nivel de percepción del consumo, c) características del consumo y se estableció además los aspectos sociodemográficos de la vida de los adolescentes.

El levantamiento de la información se realizó en las aulas de clases de las instituciones educativas con la autorización de las autoridades respectivas, los estudiantes respondieron en forma anónima y recibieron las instrucciones para complementar la información. Los datos obtenidos fueron analizados con SPSS 21.0 estimando proporciones y diferencias identificadas con la prueba de $\mathrm{x}^{2}$ Chi cuadrado y un valor de significancia de $\mathrm{p}<0,05$. La investigación se realizó de enero a diciembre del 2015. 


\section{Resultados.}

El análisis de las características sociodemográficas demuestra que el 60,37\% son mujeres y 39,63\% hombres, Figura 1. El 30,24\% tienen 15 años de edad, el 28,90\% 16 años y el 21,77\% tiene 17 años. Figura 2. Se encontró en relación a la etnia, que el 97,9\% de los adolescentes son mestizos. Se consideró además saber si trabajan y el $84,5 \%$ no lo hacen, en lo que respecta a los integrantes de la familia, el $23 \%$ tiene cinco integrantes y el $21,5 \%$ son cuatro miembros. El 60,2\% vive con sus padres, con un estatus socioeconómico medio en un 57,6\%, la religión que profesan en su mayoría $55 \%$ es la católica. Tabla 1.

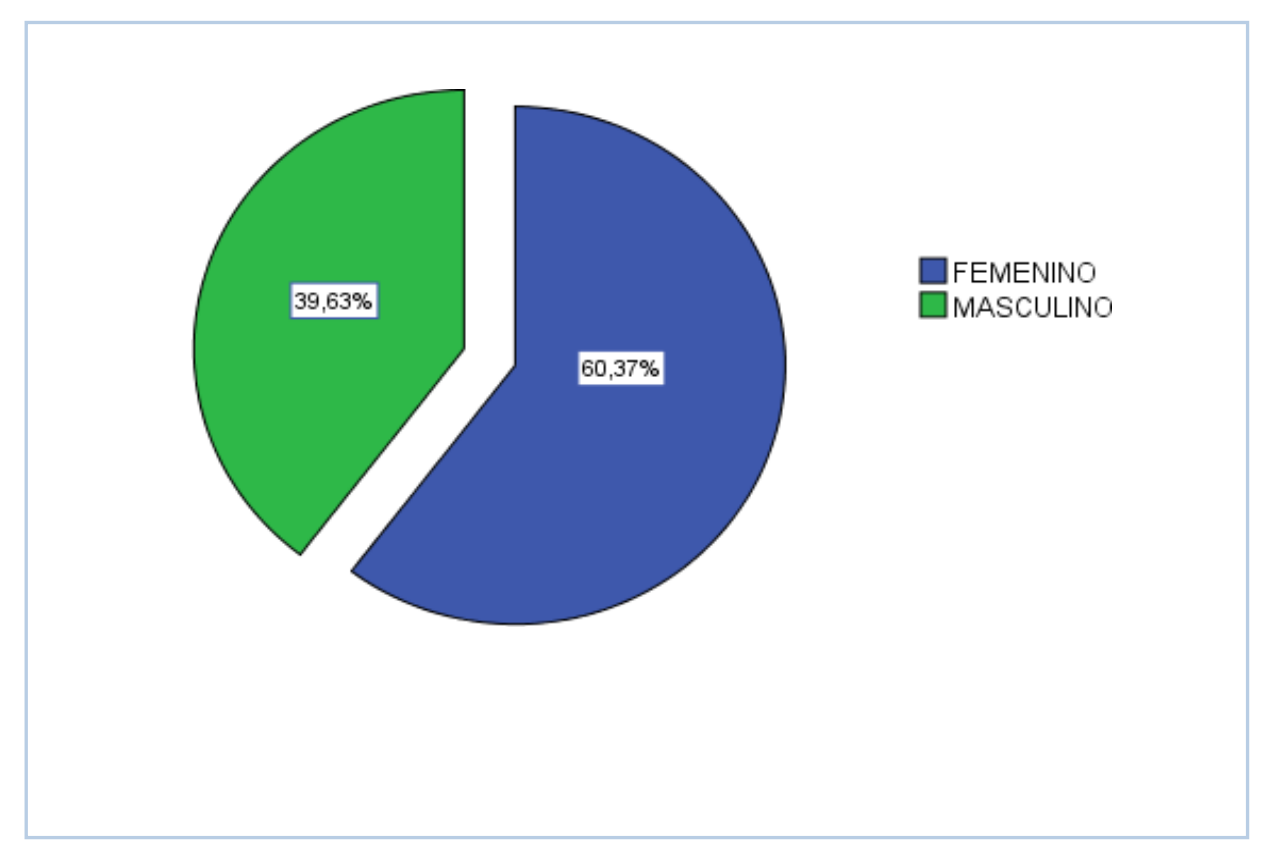

Figura 1. Género de adolescentes 


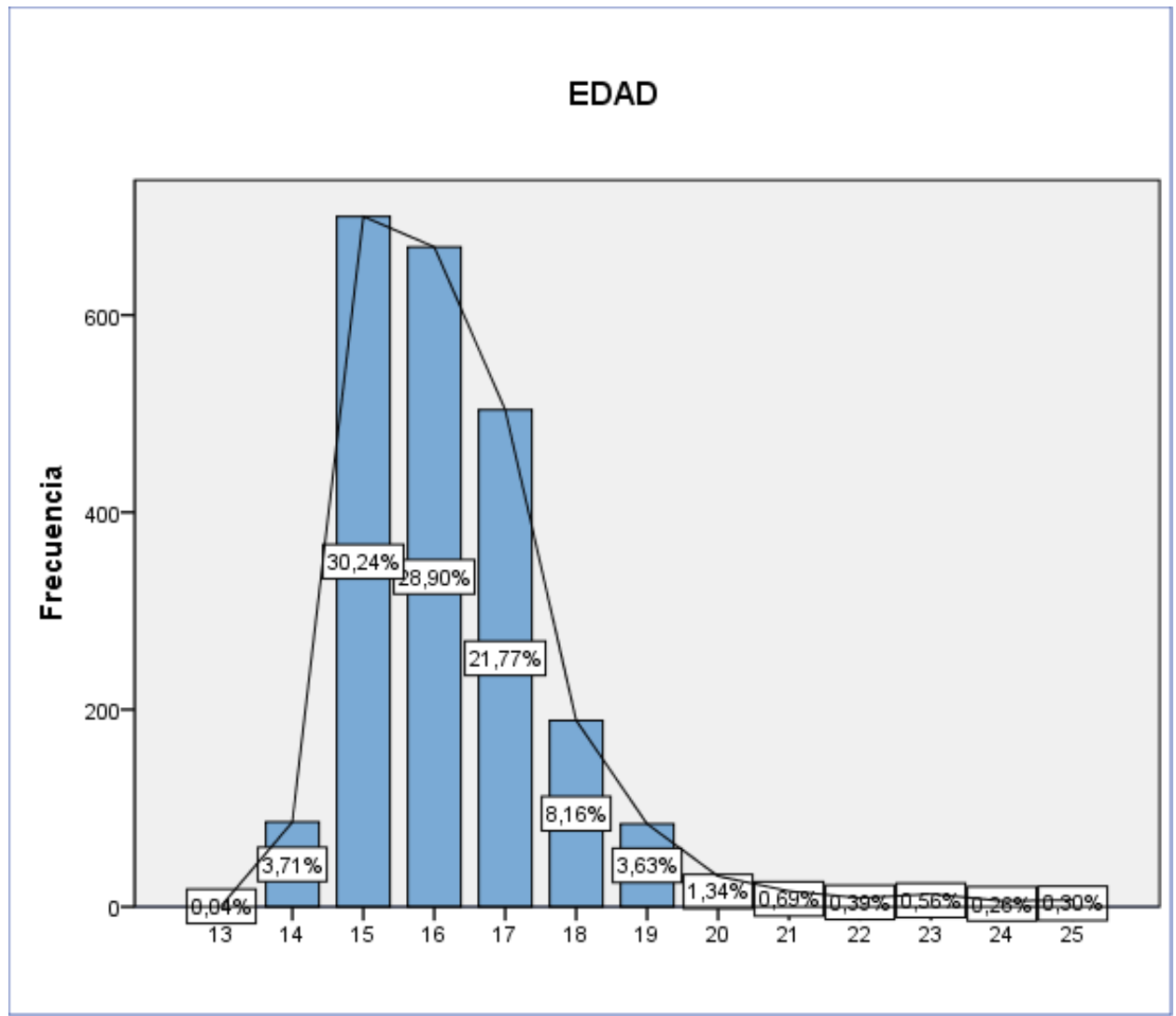

Figura 2. Frecuencia de edad de adolescentes

Tabla 1. Características sociodemográficas

\begin{tabular}{|c|c|c|}
\hline Variables & Categorías & Prevalencia \% \\
\hline Género: & $\begin{array}{l}\text { Hombre } \\
\text { Mujer }\end{array}$ & $\begin{array}{l}39,63 \\
60,37\end{array}$ \\
\hline Etnia & $\begin{array}{l}\text { Mestizo } \\
\text { Afroecuatoriano } \\
\text { Indígena } \\
\text { Blanca }\end{array}$ & $\begin{array}{l}97,9 \\
0,8 \\
0,2 \\
1,1\end{array}$ \\
\hline Trabaja & $\begin{array}{l}\text { No } \\
\text { Si }\end{array}$ & $\begin{array}{l}84,5 \\
10,3\end{array}$ \\
\hline $\begin{array}{l}\text { Integrantes de la } \\
\text { familia }\end{array}$ & $\begin{array}{l}\text { Tres } \\
\text { Cuatro } \\
\text { Cinco }\end{array}$ & $\begin{array}{l}9,8 \\
21,5 \\
23\end{array}$ \\
\hline
\end{tabular}




\begin{tabular}{|l|l|l|}
\hline & Seis & 13,1 \\
\hline Religión & Católico & 55 \\
& Evangelista & 13,5 \\
& Cristiano & 10,6 \\
& Ninguno & 16,8 \\
\hline Estructura familiar & Vive con sus & 60,2 \\
& padres & 23,8 \\
& Vive con su madre & 3,3 \\
& Vive con su padre & 8,2 \\
\hline Estatus & Otros familiares & \\
socioeconómico & Medio & 57,6 \\
& Medio bajo & 23,5 \\
& Medio Alto & 10,2 \\
\hline
\end{tabular}

Se evaluaron aspectos del estilo de vida como el hacer ejercicio: caminar, trotar, hacer deporte, la práctica de actividades de recreación: caminar, nadar, montar en bicicleta, ir al médico una vez al año, ir al odontólogo una vez al año, si se automedica o va al farmacéutico, el tipo de alimentación con nutrientes, consumo de comidas rápidas, si se mantiene un horario regular de comidas, trasnochar, si ha tenido alguna vez relaciones sexuales y si ha sido víctima de violencia en los últimos doce meses, en relación al género y a la edad, mediante $\mathrm{x}^{2}$ chi cuadrado con un $\mathrm{p}<0,05$.

Se encontró que existe una relación significativa $\mathrm{p}=0,000$ en relación al género y el hacer ejercicios, caminar, trotar o hacer deporte así como al practicar actividades de recreación caminar, nadar, montar en bicicleta, considerándose que lo realizan algunas veces $36 \%$ de estudiantes del género femenino y 41,8\% del masculino algunas veces van al médico una vez al año ( $\mathrm{p}=0,019)$. El $42,8 \%$ de las mujeres y el $32,9 \%$ de varones $(\mathrm{p}=0,013)$ van al odontólogo una vez al año algunas veces. $42,1 \%$ de mujeres y $38,9 \%$ de varones $(\mathrm{p}=0,018)$ se automedican o van al farmacéutico. En relación al tipo de alimentación existe un nivel de significancia del p=0,188 significativo en relación al tipo de alimentación que se ingiere con nutrientes en relación al género. No existe relación significativa entre el mantener un horario de comidas regular $(\mathrm{p}=0,059)$ y el trasnochar $(\mathrm{p}=0,196)$ y si ha sido víctima de violencia en los últimos doce meses $(\mathrm{p}=0,188)$, en tanto que el nivel de 
significancia es de $(\mathrm{p}=0,000)$ en el $25,7 \%$ del género femenino y 42,4\% del género masculino que han tenido alguna vez relaciones sexuales. Tabla 2.

Tabla 2. Factores asociados al estilo de vida según género ( $\mathrm{x}^{2}$ chi cuadrado)

\begin{tabular}{|c|c|c|c|c|}
\hline Variables asociadas & Género & \multicolumn{2}{|c|}{ Frecuencia } & $\begin{array}{l}\text { Nivel significación } x^{2} \\
\text { chi cuadrado } p<0,05\end{array}$ \\
\hline $\begin{array}{l}\text { Hacer ejercicio camina, } \\
\text { trota, hace deporte }\end{array}$ & $\begin{array}{l}\text { Masculino } \\
\text { Femenino }\end{array}$ & \multicolumn{2}{|c|}{$\begin{array}{l}\text { Algunas veces } \\
31,9 \% \\
52,9 \%\end{array}$} & 0,000 \\
\hline $\begin{array}{l}\text { Practica actividades de } \\
\text { recreación } \\
\text { nadar, montar en en } \\
\text { bicicleta }\end{array}$ & $\begin{array}{l}\text { Femenino } \\
\text { Masculino }\end{array}$ & \multicolumn{2}{|c|}{$\begin{array}{l}\text { Algunas veces } \\
46,6 \% \\
33 \%\end{array}$} & 0,000 \\
\hline $\begin{array}{l}\text { Ir al médico una vez al } \\
\text { año }\end{array}$ & $\begin{array}{l}\text { Femenino } \\
\text { Masculino }\end{array}$ & \multicolumn{2}{|c|}{$\begin{array}{l}\text { Algunas veces } \\
36 \% \\
41,8 \%\end{array}$} & 0,019 \\
\hline $\begin{array}{l}\text { Ir al odontólogo una vez } \\
\text { al año }\end{array}$ & $\begin{array}{l}\text { Femenino } \\
\text { Masculino }\end{array}$ & \multicolumn{2}{|c|}{$\begin{array}{l}\text { Algunas veces } \\
42,8 \% \\
32,9 \%\end{array}$} & 0,013 \\
\hline $\begin{array}{l}\text { Se automedica o va al } \\
\text { farmacéutico }\end{array}$ & $\begin{array}{l}\text { Femenino } \\
\text { Masculino }\end{array}$ & \multicolumn{2}{|c|}{$\begin{array}{l}\text { Algunas veces } \\
42,1 \% \\
38,9 \%\end{array}$} & 0,018 \\
\hline $\begin{array}{l}\text { Tipo de Alimentación } \\
\text { con nutrientes }\end{array}$ & $\begin{array}{l}\text { Femenino } \\
\text { Masculino }\end{array}$ & \multicolumn{2}{|c|}{$\begin{array}{l}\text { Siempre } \\
49,2 \% \\
45,8 \%\end{array}$} & 0,188 \\
\hline $\begin{array}{l}\text { Consume } \\
\text { rápidas }\end{array}$ & $\begin{array}{l}\text { Femenino } \\
\text { Masculino }\end{array}$ & \multicolumn{2}{|c|}{$\begin{array}{l}\text { Algunas veces } \\
58 \% \\
53,5 \%\end{array}$} & 0,049 \\
\hline $\begin{array}{l}\text { Mantiene horario } \\
\text { regular de comidas }\end{array}$ & $\begin{array}{l}\text { Femenino } \\
\text { Masculino }\end{array}$ & \multicolumn{2}{|c|}{$\begin{array}{l}\text { Algunas veces } \\
38,6 \% \\
34,7 \%\end{array}$} & 0,059 \\
\hline Trasnocha & $\begin{array}{l}\text { Femenino } \\
\text { Masculino }\end{array}$ & \multicolumn{2}{|c|}{$\begin{array}{l}\text { Algunas veces } \\
52,5 \% \\
50,3 \%\end{array}$} & 0,196 \\
\hline $\begin{array}{l}\text { Alguna vez ha tenido } \\
\text { relaciones sexuales }\end{array}$ & $\begin{array}{l}\text { Femenino } \\
\text { Masculino }\end{array}$ & $\begin{array}{l}\text { No } \\
74,1 \% \\
57 \%\end{array}$ & $\begin{array}{l}\mathrm{Si} \\
25,7 \% \\
42,4 \%\end{array}$ & 0,000 \\
\hline $\begin{array}{l}\text { Ha sido víctima de } \\
\text { violencia en los últimos } \\
\text { doce meses }\end{array}$ & $\begin{array}{l}\text { Femenino } \\
\text { Masculino }\end{array}$ & \multicolumn{2}{|c|}{$\begin{array}{l}\text { Ninguna } \\
83,5 \% \\
80,9 \%\end{array}$} & 0,188 \\
\hline
\end{tabular}


En relación a la edad y las variables del estilo de vida, se consideró el rango de edad de los 15 a los 17 años para el análisis de los resultados. Existe una relación significativa entre la edad y el hacer ejercicio caminar, trotar o hacer deporte $(\mathrm{p}=0,005)$, practicar actividades de recreación, caminar, nadar, montar en bicicleta $(\mathrm{p}=0,000)$. No existe relación entre el ir al médico una vez al año, ir al odontólogo una vez al año, automedicarse o ir al farmacéutico, el tipo de alimentación con nutrientes, mantener un horario regular de comidas y la edad. En tanto que el nivel de significancia entre la edad y el consumo de comidas rápidas es de $(\mathrm{p}=0,004)$, en la misma medida que es significativa la relación entre la edad y el trasnochar $(\mathrm{p}=0,003)$ y el haber tenido alguna vez relaciones sexuales $(\mathrm{p}=0,000)$ así como el haber sido víctima de violencia en los últimos doce meses $(\mathrm{p}=0,000)$. Tabla 3 .

Tabla 3. Factores asociados al estilo de vida según edad $\left(x^{2}\right.$ chi cuadrado)

\begin{tabular}{|c|c|c|c|}
\hline Variables asociadas & Edad & Frecuencia & $\begin{array}{l}\text { Nivel significación } \mathrm{x}^{2} \\
\text { chi cuadrado } \mathrm{p}<0,05\end{array}$ \\
\hline $\begin{array}{l}\text { Hacer ejercicio camina, } \\
\text { trota, hace deporte }\end{array}$ & $\begin{array}{l}15 \text { años } \\
16 \text { años } \\
17 \text { años }\end{array}$ & $\begin{array}{l}\text { Algunas veces } \\
45,6 \% \\
45,1 \% \\
42,9 \%\end{array}$ & 0,005 \\
\hline $\begin{array}{l}\text { Practica actividades de } \\
\text { recreación caminar, } \\
\text { nadar, montar en } \\
\text { bicicleta }\end{array}$ & $\begin{array}{l}15 \text { años } \\
16 \text { años } \\
17 \text { años }\end{array}$ & $\begin{array}{l}\text { Algunas veces } \\
40,8 \% \\
43,8 \% \\
38,4 \%\end{array}$ & 0,000 \\
\hline $\begin{array}{l}\text { Ir al médico una vez al } \\
\text { año }\end{array}$ & $\begin{array}{l}15 \text { años } \\
16 \text { años } \\
17 \text { años }\end{array}$ & $\begin{array}{l}\text { Algunas veces } \\
36,4 \% \\
36,4 \% \\
40,9 \%\end{array}$ & 0,657 \\
\hline $\begin{array}{l}\text { Ir al odontólogo una vez } \\
\text { al año }\end{array}$ & $\begin{array}{l}15 \text { años } \\
16 \text { años } \\
17 \text { años }\end{array}$ & $\begin{array}{l}\text { Algunas veces } \\
40,5 \% \\
39,5 \% \\
44,9 \%\end{array}$ & 0,383 \\
\hline $\begin{array}{l}\text { Se automedica o va al } \\
\text { farmacéutico }\end{array}$ & $\begin{array}{l}15 \text { años } \\
16 \text { años } \\
17 \text { años }\end{array}$ & $\begin{array}{l}\text { Algunas veces } \\
41 \% \\
41,2 \% \\
39,1 \%\end{array}$ & 0,984 \\
\hline
\end{tabular}




\begin{tabular}{|c|c|c|c|c|}
\hline $\begin{array}{l}\text { Tipo de Alimentación } \\
\text { con nutrientes }\end{array}$ & $\begin{array}{l}15 \text { años } \\
16 \text { años } \\
17 \text { años }\end{array}$ & \multicolumn{2}{|c|}{$\begin{array}{l}\text { Siempre } \\
35,7 \% \\
51,7 \% \\
47,6 \%\end{array}$} & 0,849 \\
\hline $\begin{array}{l}\text { Consume comidas } \\
\text { rápidas }\end{array}$ & $\begin{array}{l}15 \text { años } \\
16 \text { años } \\
17 \text { años }\end{array}$ & \multicolumn{2}{|c|}{$\begin{array}{l}\text { Algunas veces } \\
55,7 \% \\
56,5 \% \\
59,8 \%\end{array}$} & 0,004 \\
\hline $\begin{array}{l}\text { Mantiene horario } \\
\text { regular de comidas }\end{array}$ & $\begin{array}{l}15 \text { años } \\
16 \text { años } \\
17 \text { años }\end{array}$ & \multicolumn{2}{|c|}{$\begin{array}{l}\text { Algunas veces } \\
36,8 \% \\
37 \% \\
36,6 \%\end{array}$} & 0,284 \\
\hline Trasnocha & $\begin{array}{l}15 \text { años } \\
16 \text { años } \\
17 \text { años }\end{array}$ & \multicolumn{2}{|c|}{$\begin{array}{l}\text { Algunas veces } \\
51,2 \% \\
52,7 \% \\
52,4 \%\end{array}$} & 0,003 \\
\hline $\begin{array}{l}\text { Alguna vez ha tenido } \\
\text { relaciones sexuales }\end{array}$ & $\begin{array}{l}15 \text { años } \\
16 \text { años } \\
17 \text { años }\end{array}$ & $\begin{array}{l}\text { No } \\
78,9 \% \\
69,7 \% \\
64,5 \%\end{array}$ & $\begin{array}{l}\mathrm{Si} \\
20,8 \% \\
29,8 \% \\
35,1 \%\end{array}$ & 0,000 \\
\hline $\begin{array}{l}\text { Ha sido víctima de } \\
\text { violencia en los últimos } \\
\text { doce meses }\end{array}$ & $\begin{array}{l}15 \text { años } \\
16 \text { años } \\
17 \text { años }\end{array}$ & \multicolumn{2}{|c|}{$\begin{array}{l}\text { Ninguna } \\
82,9 \% \\
83,1 \% \\
83,3 \%\end{array}$} & 0,000 \\
\hline
\end{tabular}

Respecto al género $(\mathrm{p}=0,049)$ y la edad $(\mathrm{p}=0,036)$ tienen una correlación con el consumo de licor al menos dos veces al mes, con un nivel de significancia de $\mathrm{p}<0,05$. Se encontró que el género y la edad se relacionan significativamente $(\mathrm{p}=0,000)$ en correspondencia al consumo de alcohol, con un nivel de significancia de $\mathrm{p}<0,05$, tanto en la cantidad de bebidas ingeridas por día, el número de días, la cantidad de veces que se embriagó el adolescente así como la forma en que consiguió las bebidas alcohólicas. Tabla 4. 
Tabla 4. Condiciones del consumo de alcohol según el género y la edad

\begin{tabular}{|c|c|c|c|c|}
\hline \multicolumn{2}{|l|}{ Variables asociadas } & \multicolumn{2}{|l|}{ Frecuencia } & \multirow{2}{*}{$\begin{array}{l}\text { Nivel significación } \mathbf{x}^{2} \\
\text { chi cuadrado } \mathbf{p}<\mathbf{0 , 0 5}\end{array}$} \\
\hline $\begin{array}{l}\text { Cuántas bebidas } \\
\text { ingirió por día }\end{array}$ & $\begin{array}{l}\text { Género: } \\
\text { Masculino } \\
\text { Femenino }\end{array}$ & $\begin{array}{l}1 \text { bebida } \\
8,9 \% \\
6,9 \%\end{array}$ & $\begin{array}{l}2 \text { bebidas } \\
4,8 \% \\
5,0 \%\end{array}$ & \\
\hline & $\begin{array}{l}\text { Edad: } \\
15 \text { años } \\
16 \text { años } \\
17 \text { años }\end{array}$ & $\begin{array}{l}1 \text { bebida } \\
6,4 \% \\
6,4 \% \\
9,0 \%\end{array}$ & $\begin{array}{l}2 \text { bebidas } \\
4,2 \% \\
3,7 \% \\
6,6 \%\end{array}$ & 0,000 \\
\hline \multirow{2}{*}{$\begin{array}{lc}\text { Cuántos días } \\
\text { ingirió } & \text { una } \\
\text { bebida } & \text { que } \\
\text { contenía alcohol }\end{array}$} & $\begin{array}{l}\text { Género } \\
\text { Masculino } \\
\text { Femenino }\end{array}$ & $\begin{array}{l}1 \text { a } 2 \text { días } \\
17,3 \% \\
14,4 \%\end{array}$ & $\begin{array}{l}3 \text { a } 5 \text { días } \\
4,0 \% \\
2,4 \%\end{array}$ & 0,000 \\
\hline & $\begin{array}{l}\text { Edad: } \\
15 \text { años } \\
16 \text { años } \\
17 \text { años }\end{array}$ & $\begin{array}{l}1 \text { a } 2 \text { días } \\
12,5 \% \\
14,4 \% \\
16,0 \%\end{array}$ & $\begin{array}{l}3 \text { a } 5 \text { días } \\
1,7 \% \\
2,5 \% \\
2,6 \%\end{array}$ & 0,000 \\
\hline \multirow[t]{2}{*}{$\begin{array}{l}\text { Cuántas veces se } \\
\text { embriagó }\end{array}$} & $\begin{array}{l}\text { Género: } \\
\text { Masculino } \\
\text { Femenino }\end{array}$ & $\begin{array}{l}1 \text { a } 2 \text { veces } \\
21,5 \% \\
15,4 \%\end{array}$ & $\begin{array}{l}3 \text { o } 9 \text { veces } \\
4,7 \% \\
3,9 \%\end{array}$ & 0,000 \\
\hline & $\begin{array}{l}\text { Edad: } \\
14 \text { años } \\
15 \text { años } \\
16 \text { años }\end{array}$ & $\begin{array}{l}1 \text { a } 2 \text { veces } \\
11,8 \% \\
15,8 \% \\
20,2 \%\end{array}$ & $\begin{array}{l}3 \text { o } 9 \text { veces } \\
3,0 \% \\
4,1 \% \\
4,7 \%\end{array}$ & 0,000 \\
\hline \multirow{2}{*}{$\begin{array}{l}\text { Como consiguió } \\
\text { el alcohol que } \\
\text { bebió }\end{array}$} & $\begin{array}{l}\text { Género: } \\
\text { Masculino } \\
\text { Femenino }\end{array}$ & $\begin{array}{l}\text { En una } \\
\text { tienda } \\
45,8 \% \\
49,2 \%\end{array}$ & $\begin{array}{l}\text { Con } \quad \text { los } \\
\text { amigos } \\
8,3 \% \\
7,6 \%\end{array}$ & 0,000 \\
\hline & $\begin{array}{l}\text { Edad: } \\
15 \text { años } \\
16 \text { años } \\
17 \text { años }\end{array}$ & $\begin{array}{l}\text { En } \\
\text { tienda } \\
3,3 \% \\
3,7 \% \\
6,4 \%\end{array}$ & $\begin{array}{l}\text { Con } \quad \text { los } \\
\text { amigos } \\
5,4 \% \\
8,4 \% \\
8,2 \%\end{array}$ & 0,000 \\
\hline
\end{tabular}

La media de edad al consumir licor al menos dos veces a la semana es casi siempre a los 17 años y siempre a los 16 años. Los resultados expresan que 894 estudiantes del género femenino consumieron alcohol entre 6 a 9 días, 875 consumieron entre 20 a 29 días y en el género masculino 
935 consumieron alcohol entre 20 a 29 días y 290 consumieron entre 10 a 19 días, con un p=0,000.

Figura 3.

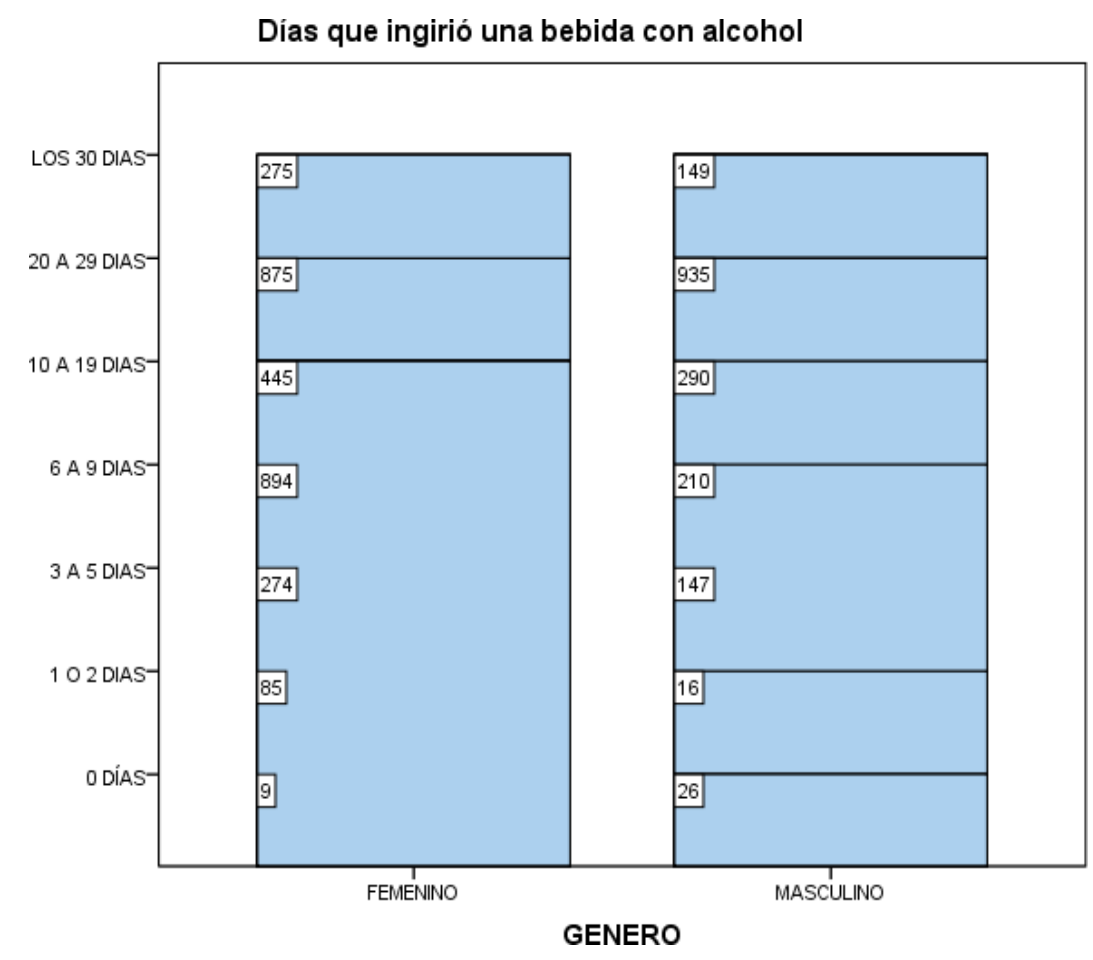

Figura 3. Consumo de alcohol según días y género

\section{Discusión.}

Los resultados del estudio evidencian que el estilo de vida de los adolescentes no es saludable, considerándose el hecho de estar en pleno proceso de maduración tanto físico como intelectual. En los últimos años se ha producido entre los adolescentes un cambio en el concepto de ocio y tiempo libre, lo cual, unido a desajustes afectivos, psicosociales y educativos propios de esta edad, podría tener relación con el desarrollo de conductas de riesgo (Paniagua, García, Castellano, Sarrallé y Redondo, 2001). 
Se encuentra que las adolescentes son quienes en mayor porcentaje realizan ejercicios como caminar, trotar o hacer deportes, así como practicar actividades de recreación, en la edad comprendida entre los 14 a 16 años. Estudios como el de Balaguer (2002) manifiestan que la práctica de la actividad física es una conducta del adolescente que favorece el desarrollo de estilos de vida saludables. En tanto que Huescar, Cervello, Llamas, et al, (2011) expresan que la inclusión de actividades físico-deportivas en la rutina de vida en la adolescencia contribuiría a la adopción de un modelo de vida saludable, al garantizar la prevención y extinción de otras conductas no saludables, tales como el consumo de tabaco, alcohol y otras drogas.

El consumo de comidas rápidas se presenta de manera frecuente poniéndose de manifiesto que las mujeres ingieren en mayor proporción este tipo de alimentos que los hombres, asociado a las edades de mayor frecuencia de consumo comprendidas de los 14 a 16 años. Jiménez (2011) indica que los hábitos alimentarios nocivos se presentan como un elemento de riesgo para la salud, la alimentación con sustancias nutritivas es indispensable para la salud y poder efectuar las actividades diarias con vitalidad y sin cansancio acorde a esta etapa de la vida.

El consumo de licor se produce a una edad de 16 años al menos dos veces a la semana. El alcohol es la droga más consumida por los adolescentes y presenta un patrón de consumo esporádico, intensivo, fuera del hogar y con sus pares (Paniagua, García, et al, 2001). Los adolescentes del género masculino ingieren 1 o 2 bebidas por día y consiguen las bebidas alcohólicas en la tienda o con los amigos por él fácil acceso a los licores.

El consumo de alcohol generalmente está relacionado con estilos de vida de riesgo como: consumo de tabaco y uso de drogas ilegales; trastornos mentales, como la depresión y la ansiedad; desórdenes alimenticios y aumento del índice de masa corporal; y, también, las peleas en la escuela, 
acoso, vandalismo y otros tipos de violencia. (Malta, Sardinha, Mendes, Barreto, Giatti, Castro, et al., 2009).

De acuerdo a Cava, Mugui y Musitu (2008) la edad tiene efectos como factor de riesgo y de protección en la adolescencia temprana y media en el consumo de sustancias. Los adolescentes aún no tienen la capacidad de anticipar los peligros de un comportamiento habitual, que los hace más vulnerables a la participación en las conductas riesgo, como el uso y abuso de sustancias, especialmente cuando son más agresivos e impulsivos (Kazdin, 1993).

La adolescencia es una etapa difícil que genera vulnerabilidad, donde se producen conflictos y adaptaciones en el entorno sociocultural, Los adolescentes que consumen bebidas alcohólicas tienden a "probar y experimentar" el consumo de otras drogas. Paniagua, García, Castellano, Sarrallé y Redondo (2001) indican que en el consumo de tabaco y alcohol como drogas destacan como factores de riesgo la influencia del entorno familiar y social del consumidor, asociados a patrones de ocio, presencia de episodios de embriaguez y consumo de varias drogas entre los adolescentes.

\section{Conclusiones.}

Los hallazgos de la investigación permitieron conocer que a una edad de 16 a 17 años se consume licor lo cual afecta la salud como la calidad de vida de este importante grupo de la población. Se encontró además que no existen diferencias significativas entre el consumo de hombres y mujeres, lo cual pudiera darse por la gran libertad que existe en la actualidad lo que expone en gran medida al riesgo del consumo de bebidas alcohólicas. La adolescencia es un período de grandes cambios, caracterizado por nuevos hábitos y exposición a factores de riesgo como tabaquismo, dieta inadecuada, sedentarismo (Carvalho, Medeiros, López, Barreto y Morais, 2014). 
Los adolescentes tienen hábitos de vida no saludables como una escasa práctica de ejercicios físicos, actividades de recreación, hábitos alimenticios inadecuados, en relación a la salud física y mental que conducen al consumo de alcohol y tabaco.

La edad, el sexo, las condiciones de vida a las que se ve expuesto un adolescente son condicionantes del consumo de sustancias legales, por su fácil acceso en el medio social en donde se desenvuelven. Los hábitos de comportamiento tanto saludables como no saludables que tienen los adolescentes en su vida cotidiana influyen de manera significativa en el desarrollo del consumo de sustancias adictivas como alcohol.

El alcohol es la droga más consumida por los adolescentes y presenta un patrón de consumo esporádico, intensivo, fuera del hogar y con sus pares (Paniagua, García, et al, 2001). La conducta desinhibida que induce el consumo de alcohol y de drogas ilegales facilita las relaciones sexuales precoces y en consecuencia las enfermedades de transmisión sexual y los embarazos no deseados (Miret, Rodes, Valverde, Geli y Cassabona, 1997). De acuerdo a Buscemi y Turchi (2011) la edad de inicio al beber y antecedentes familiares de problemas con el alcohol son dos de los factores de riesgo más importantes de inicio del consumo.

Paniagua, García, Castellano, Sarrallé y Redondo (2001) afirman: “en la actualidad, se observa que los adolescentes contactan de forma precoz con tabaco, alcohol y drogas no legales y muchos de ellos presentan un alto consumo de estas sustancias". Es importante señalar que el consumir drogas como el alcohol y tabaco es perjudicial para la salud, siendo el riesgo de enfermedad y muerte mucho mayor cuando se suman ambas adicciones. Y se debe considerar que: el inicio temprano de fumar y beber aumenta el riesgo de uso posterior de cannabis u otras drogas (Becoña, et al, 2011). 
Los patrones patológicos de consumo de alcohol en el adulto, iniciados en muchos casos durante la adolescencia, pueden ocasionar cirrosis hepática, enfermedades cardiovasculares y neurológicas. (Paniagua, García, Castellano, Sarrallé y Redondo, 2001).

El consumo esporádico de alcohol es más común en los adolescentes y pueden llegar al abuso ocasionando riesgos potenciales para la salud como intoxicación, además el consumo de alcohol en la adolescencia puede producir accidentes de tráfico, homicidios suicidios, que representan las principales causas de muerte entre los jóvenes (Modelli, Pratesi, Tauil, 2008).

Por consiguiente, se debe realizar promoción y prevención de la salud que permita concientizar en los/as adolescentes los efectos nocivos que ocasionan el consumo de esta sustancia. Así como fomentar la importancia de la práctica de estilos de vida saludables que conlleven a mejorar la calidad de vida de este grupo vulnerable de la población.

\section{Agradecimiento.}

En especial a los estudiantes participantes en la investigación por su gran espíritu de colaboración, así como a los directores de las instituciones educativas. Mi más sincero agradecimiento a cada uno de ustedes.

\section{Referencias bibliográficas}

Álvarez, E., Fraile, A, M., Secades, R. y otros (2005). Percepción de riesgo del consumo de drogas en escolares de enseñanza secundaria del principado de Asturias. España: Colegio Oficial de Psicólogos de Asturias

Ariza. C. y Nebot, M. (2002). Predictores de la iniciación al consumo de tabaco en escolares de enseñanza secundaria de Barcelona y Lleida. Revista Española de Salud Pública, 76, 3, 22738.

Balaguer, I. (2002). Estilos de vida en la adolescencia. Valencia: Promolibro. 
Becoña, E., López, A., Fernández del Río, E., Martínez, U., Osorio, J., Fraga, J., Arrojo, M., López, F., Domínguez, MN. (2011). Drunkness, driving and sexual relations in young cocaine and ecstasy users. Adicciones, 23, 205-218.

Buscemi L, Turchi C. (2011). An overview of the genetic susceptibility to alcoholism. Med Sci Law 51:S2-6. doi: 10.1258/msl.2010.010054.

Carvalho, D., Medeiros, M., López, D., Barreto, S. y Morais, O. (2014). Exposure to alcohol among adolescent students and associated factors. Revista de salud pública, 48, 52-62.

Cava, M.J., Mugui, S., y Musitu, G. (2008). Diferencias en factores de protección del consumo de sustancias en la adolescencia temprana y media. Psicothema, 20, 389-395.

Consep. (2014). Cuarta encuesta nacional sobre uso de drogas en estudiantes de 12 a 17 años. Recuperado de: http://sisco.copolad.eu/web/uploads/documentos/Encuesta_uso_drogas_CONSEP_08.2013.pd $\mathrm{f}$

Cicua, D., Méndez, M. y Muñoz, L. (2008). Factores en el consumo de alcohol en adolescentes. Pensamiento Psicológico, 4, 15-134.

Henderson, J.B, Hall, S. M. y Lipton, H. L. (1980). Changing selfdestructive behaviors. Health Psychology. San Francisco: Jossey Bass.

Huescar, E., Cervello, E., Llamas, L. y Moreno, J. (2011). Alcohol and tobacco consumption behaviors and their relationship to healthy habits in adolescents. Behavioral PsychologyPsicología Conductual, 19, 523-539.

Instituto Nacional de Estadísticas y Censos. (2012). Ecuador en cifras. Recuperado de: http://www.ecuadorencifras.gob.ec/documentos/webinec/Revistas/Postdata/postdata01/files/as sets/downloads/page0006.pdf

Jiménez, T. (2011). Autoestima de riesgo y protección: una mediación entre el clima familiar y el consumo de sustancias en adolescentes. Intervención Psicosocial: Revista sobre Igualdad y Calidad de Vida, 20, 53-61.

Kazdin, A. E. (1993). Adolescent mental health: Prevention and treatment programs. American Psychologist, 48, 127-141.

Malta, DC., Porto, DL., Melo, FC., Monteiro, RA., Sardinha, LM., Lessa, BH. (2011). Família e proteção aouso de tabaco, álcool e drogas em adolescentes, Pesquisa Nacional de Saúde dos Escolares. Revista Brasilera Epidemiología, 14, 166-77.

Malta, DC., Sardinha, LM., Mendes, I., Barreto, SM., Giatti, L., Castro, IR, et al. (2009). Prevalência de fatores de risco e proteção de doenças crônicas não transmissíveis em adolescentes: resultados da Pesquisa Nacional de Saúde do Escolar (PeNSE), Brasil, 2009. Ciencia Salud Colectiva, 15, 9-19. 
Miret, M., Rodes, A., Valverde, G., Geli, M., Cassabona, J. (1997). Conductas de riesgo relacionadas con infección por el virus de inmunodeficiencia humana en adolescentes escolarizados en Cataluña. Gaceta Sanitaria, 11, 66-73.

Modelli, M., Pratesi, R. y Tauil, PL. (2008). Blood alcohol concentration in fatal traffic accidents in the Federal District, Brazil. Revista Saude Pública. 42(2), 350-2. DOI:10.1590/S003489102008005000012

Muñoz, M. J. y Graña, J. L. (2001). Factores familiares de riesgo y de protección para el consumo de drogas en adolescentes. Psicothema, 13, 1, 87-94.

Organización Mundial de la Salud. (2014). Informe consumo alcohol y tabaco en el mundo.

Recuperado de:

http://apps.who.int/iris/bitstream/10665/112736/1/9789240692763_eng.pdf?ua=1

Organización Panamericana de la Salud. (2012). Salud en las Américas. Recuperado de:

http://www.paho.org/saludenlasamericas/index.php?option=com_content $\& v i e w=a r t i c l e \& i d=9$ \&Itemid=14\&lang=es

Organización Panamericana de la Salud. (2018). Alcohol. Recuperado de:

http://www.who.int/es/news-room/fact-sheets/detail/alcohol

OPS/OMS. (2014). Ecuador. Recuperado de:

https://www.paho.org/ecu/index.php?option=com_content\&view=article\&id=1208:mayo-14$2014 \&$ Itemid $=972$

Paniagua, HP., García, SG., Castellano, GC., Sarrallé, R. y Redondo, C. (2001). Tobacco, alcohol and illegal drug consumption among adolescents. Relationship with lifestyle and environment. Anales españoles de pediatría, 55, 121-128.

Peñafiel. E. (2009). Factores de riesgo y de protección en el consumo de sustancias en adolescentes. Pulso, 32, $147-173$.

Ruiz, F. y Ruiz, J. (2011). Variables predictoras del consumo de alcohol entre adolescentes españoles. Anales de Psicología, 27, 350 - 359.

Salazar, I. y Arrivillaga, M. (2004). El consumo de alcohol, tabaco y otras drogas como parte del estilo de vida de jóvenes universitarios. Revista colombiana de Psicología, 13, 74 - 89.

Tegoma-Ruiza, V.M. y Cortaza-Ramírez, L. (2016). Prevalencia del consumo de alcohol en adolescentes de una secundaria de Coatzacoalcos, Veracruz. Enfermería. Universitaria, 13, 4.

Tirado, R., Aguaded, J. I. y Marin, I. (2009). Factores de protección y de riesgo del consumo de alcohol en alumnos de la Universidad de Huelva. Salud y Drogas, 9, 165-184. 